\title{
Incidência de glaucoma agudo primário no Serviço de Glaucoma do Hospital São Geraldo
}

\author{
Incidence of primary angle-closure glaucoma in the Glaucoma Service of the \\ São Geraldo Hospital
}

\author{
Rafael Vidal Mérula ${ }^{1}$ \\ Sebastião Cronemberger ${ }^{2}$ \\ Nassim Calixto ${ }^{3}$
}

\begin{tabular}{|c|}
\hline RESUMO \\
\hline $\begin{array}{l}\text { Objetivos: Determinar a incidência do glaucoma agudo primário no Serviço } \\
\text { de Glaucoma do Hospital São Geraldo; estabelecer o perfil destes pacientes } \\
\text { e identificar possíveis fatores de risco. Métodos: Estudo transversal. Período } \\
\text { de análise: setembro/2005 a agosto/2006. Inclusão: diagnóstico de glaucoma } \\
\text { agudo primário. Exclusão: presença de catarata que acarrete baixa acuidade } \\
\text { visual ou miopização, glaucomas secundários, íris em platô. Foram ava- } \\
\text { liados: número de atendimentos, incidência de glaucoma agudo primário, } \\
\text { idade, sexo, raça, história familiar de glaucoma, ceratometria, e dados } \\
\text { biométricos. Resultados: Dentre } 879 \text { pacientes atendidos, } 20 \text { (2,3\%) tive- } \\
\text { ram o diagnóstico de glaucoma agudo primário, desse modo, a incidência } \\
\text { de glaucoma agudo primário foi de } 22,7 \text { por } 1000 \text { atendimentos. Dos } \\
\text { pacientes com glaucoma agudo primário: } 6(30,0 \% \text { ) eram do sexo masculino } \\
\text { e } 14(70,0 \% \text { ) feminino; aidade variou de } 40 \text { a } 73 \text { anos (média: } 60,4 \pm 8,1 \text { anos); } \\
12 \text { (60,0\%) eram leucodérmicos e } 8 \text { (40,0\%) feodérmicos; } 5 \text { (25,0\%) com } \\
\text { históriafamiliarpositiva para glaucoma. Orisco relativo para o sexofeminino } \\
\text { foi de } 1,44 \text { (IC 95\%). Onze ( } 55,0 \% \text { ) pacientes tiveram glaucoma agudo } \\
\text { primário no olho direito e } 9 \text { ( } 45,0 \% \text { ) no esquerdo. Não houve diferença } \\
\text { estatisticamente significativa na comparação dos parâmetros biométricos } \\
\text { e ceratometria entre os olhos afetados e os contralaterais. Conclusões: A } \\
\text { incidência de glaucoma agudo primário no Serviço de Glaucoma do Hospital } \\
\text { São Geraldo foi de } 22,7 \text { por } 1000 \text { atendimentos, sendo mais freqüente em } \\
\text { mulheres, leucodérmicas, com história familiar negativa para glaucoma e } \\
\text { média de idade de } 60,4 \text { anos. Os olhos afetados e olhos contralaterais foram } \\
\text { semelhantes nos parâmetros biométricos. }\end{array}$ \\
\hline
\end{tabular}

Descritores: Glaucoma de ângulo fechado/cirurgia; Glaucoma de ângulo fechado/epidemiologia; Biometria; Fatores de risco; Estudo transversal; Estudo comparativo

\section{INTRODUÇÃO}

O glaucoma é uma das principais causas de perda visual. Estima-se que 22,5 milhões de pessoas são portadoras dessa doença ${ }^{(1-2)}$. O glaucoma primário de ângulo fechado (GPAF) também chamado glaucoma agudo primário (GAP) é mais comum em países asiáticos que nos países ocidentais, e pode ser responsável por casos de cegueira se não for tratado adequadamente $^{(3-5)}$. Estudos realizados na China verificaram que o GPAF é responsável por 1,6 milhões de casos de cegueira, enquanto que o glaucoma primário de ângulo aberto (GPAA) por 0,16 milhões de $\operatorname{casos}^{(3)}$. Estudos do impacto do GPAF sobre a saúde ocular das populações ocidentais, particularmente no Brasil, ainda são raros. 
A prevalência do glaucoma depende de muitos fatores, incluindo, etnicidade, idade, sexo e região geográfica ${ }^{(3)}$. Além disso, diferenças nos instrumentos diagnósticos e metodologias para detectar a doença podem influenciar os achados epidemiológicos. A prevalência do GPAF já foi analisada em alguns estudos: 0,6\% no Nordeste da Itália ${ }^{(4)}, 1,4 \%$ na Mongólia ${ }^{(6)}, 1,0 \%$ em Cingapura ${ }^{(7)}, 0,6 \%$ no Japão ${ }^{(3)}, 0,5 \%$ a $1,08 \%$ na Índia ${ }^{(8-9)}$, $0,97 \%$ in Ponza, Itália ${ }^{(10)}$, e $0,1 \%$ em Melbourne, Austrália ${ }^{(11)}$.

Deve-se ter cuidado ao analisar os dados de prevalência, uma vez que diferentes definições de GPAF foram utilizadas. $\mathrm{O}$ Estudo Andhra Pradesh realizado no sudeste da Índia encontrou uma prevalência de $1,08 \%$ e definiu o GPAF como pressão intra-ocular (Po) maior ou igual que $22 \mathrm{mmHg}$ ou dano glaucomatoso no nervo óptico na presença de seio camerular oclusível $^{(8)}$. A prevalência do GPAF em chineses residentes na região urbana de Cingapura foi de $1,0 \%$, sendo que o glaucoma foi definido como presença de neuropatia óptica glaucomatosa e seio camerular oclusível, no qual o trabeculado pigmentado era identificado em menos de $90^{\circ}$ de circunferência ${ }^{(7)}$. Na Mongólia, a prevalência de seio oclusível, definido como uma malha trabecular que não era visível por mais de $3 / 4$ da circunferência angular na posição primária, ou GPAF, definido como seio oclusível e aumento da Po e/ou dano óptico glaucomatoso, foram $6,4 \%$ ou $1,4 \%$, respectivamente ${ }^{(6)}$. Em regiões do ocidente e Oceania, já foi estudada também a prevalência do GPAF: 0,6\% no Nordeste da Itália ${ }^{(4)}, 0,97 \%$ em Ponza, Itália ${ }^{(10)}$, e $0,1 \%$ em Melbourne, Austrália ${ }^{(11)}$; geralmente, nesses locais, a prevalência tende a ser menor que em países asiáticos. No Brasil, ainda não existem estudos definindo a prevalência do GPAF.

A fase aguda sintomática do GAP é, geralmente, de duração limitada, dessa forma, a prevalência (a freqüência de uma doença em um tempo determinado) é pouco útil como um índice de morbidade quando comparada à incidência (novos casos que ocorrem na população previamente sem a doença) ${ }^{(12)}$. A dor ocular, a baixa acuidade visual e os outros sintomas fazem com que os pacientes procurem atendimento médico quando sofrem uma crise de $\mathrm{GAP}^{(12)}$.

A incidência do GAP já foi analisada em alguns estudos que encontraram os seguintes resultados: 3,8 casos/100000, no período de 1973 a 1982, na Finlândia( ${ }^{(13)} ; 12,2 / 100000$, de março/1995 a fevereiro/1996, em Cingapura ${ }^{(12)} ; 8,3 / 100000 \mathrm{em}$ indivíduos acima de 40 anos, no período de 1980 a 1992, Olmsted County, Minesota, EUA (sendo que neste estudo avaliou-se a incidência do GPAF) ${ }^{(14)} ; 10,4 / 100000$, de março/ 1998 a fevereiro/2000, na população chinesa de Hong Kong ${ }^{(15)}$; 2,9/100000, janeiro/1995 a dezembro/2001, em Dalmácia, sudeste da Croácia ${ }^{(16)}$; 4,1/100000, 1985 a 1999, em Split, Croá$\operatorname{cia}^{(17)} ; 3,8 / 100000$, em Savoie, França ${ }^{(18)}$. Nos países ocidentais, os dados disponíveis sobre a incidência do GAP são escassos, e nenhum estudo epidemiológico, ao que saibamos, foi conduzido especificamente com este objetivo no Brasil.

Dados ecobiométricos de casos unilaterais de GAP já foram analisados em alguns estudos, nos quais avaliaram-se comparativamente o olho afetado e o olho contralateral ${ }^{(19-21)}$.
Nesses trabalhos não foram identificadas diferenças estatisticamente significativas entre os olhos nos seguintes parâmetros: câmara anterior, diâmetro axial, espessura do cristalino e relação cristalino/diâmetro axial ${ }^{(19-21)}$.

O presente estudo é o primeiro no Brasil que avalia a incidência do GAP em serviço de glaucoma de referência, além de analisar o perfil destes pacientes e possíveis fatores de risco.

O objetivo é determinar a incidência do GAP no Serviço de Glaucoma do Hospital São Geraldo (SGHSG) no período de setembro de 2005 a agosto de 2006, bem como estabelecer o perfil desses pacientes e identificar os possíveis fatores de risco. Além disso, realizar uma análise comparativa entre o olho que sofreu a crise e o olho contralateral através da avaliação dos seguintes parâmetros: ceratometria média, profundidade central média da câmara anterior, diâmetro axial médio, espessura média do cristalino e relação espessura média do cristalino e diâmetro axial médio.

\section{MÉTODOS}

Foi realizado um estudo transversal no qual foram avaliados pacientes admitidos com diagnóstico de GAP no SGHSG, dentre todos os atendimentos realizados (primeiras consultas ambulatoriais e urgência), no período de setembro de 2005 a agosto de 2006. Foram excluídos os pacientes que já haviam sido matriculados no SGHSG antes de setembro de 2005 e que estavam em acompanhamento ambulatorial. Adotaram-se os seguintes critérios de inclusão: pacientes admitidos no SGHSG com o diagnóstico de GAP. Esse diagnóstico foi definido pelos seguintes sintomas súbitos e graves apresentados pelos pacientes: dor ocular, baixa da visão com ou sem visão de arcos irisados, cefaléia, náuseas e vômitos; pressão intraocular muito elevada (maior que $35 \mathrm{mmHg}$ ) e seio camerular fechado ao exame gonioscópico no olho afetado pela crise, sendo este fechamento aposicional ou sinequial, na ausência de outras causas conhecidas de fechamento angular. Foram utilizados como critérios de exclusão: presença de catarata, exceto "glaukonfleken", à biomicroscopia que acarretasse baixa acuidade visual ou miopização; glaucomas secundários (uveítico, traumático, neovascular, afácico, pesudofácico, facomórfico ou facoanafilático) e síndrome da íris em platô. Foram analisados os seguintes parâmetros: número de atendimentos (pacientes admitidos como urgência e primeira consulta ambulatorial) realizados no SGHSG e número de casos novos de GAP diagnosticados (incidência) no período do estudo (um ano), além disso, analisaram-se alguns dados relativos aos pacientes com GAP: idade, sexo, raça, história familiar de glaucoma, ceratometria, ecobiometria (diâmetro axial, espessura do cristalino, relação espessura do cristalino e diâmetro axial). Realizou-se também uma avaliação comparativa entre o olho que teve a crise e o olho contralateral através dos dados ecobiométricos. Os dados foram analisados através do programa estatístico SPSS 8.0. 


\section{RESULTADOS}

No período de setembro de 2005 a agosto de 2006 foram realizados 879 atendimentos, sendo que $657(74,2 \%)$ foram primeiras consultas ambulatoriais e $227(25,8 \%)$ casos de urgência; foram atendidos $543(61,8 \%)$ pacientes do sexo feminino e $336(38,2 \%)$ do sexo masculino. Dentre todos os atendimentos, 20 (2,28\%) pacientes tiveram o diagnóstico de GAP, desse modo, verificou-se uma incidência de 22,7 casos novos de GAP por 1000 atendimentos no SGHSG.

Os pacientes com diagnóstico de GAP apresentavam idade variando de 40 a 73 anos com média de 60,4 anos; sendo que $14(70 \%)$ eram do sexo feminino e $6(30 \%)$ do sexo masculino; 12 (60\%) eram leucodérmicos e 8 (40\%) feodérmicos; $11(55,0 \%)$ pacientes tiveram GAP no olho direito e $9(45,0 \%)$ no olho esquerdo; 5 (25\%) pacientes tinham história positiva para glaucoma, entretanto, nenhum paciente relatava história familiar de GAP (Tabela 1). A incidência de GAP calculada para o sexo masculino era de $17,9 / 1000$ e para o sexo feminino $25,8 / 1000$, desse modo, o risco relativo para o sexo feminino era de 1,44 (intervalo de confiança de $95 \%$ ).

Nos olhos com GAP os dados ecobiométricos foram: ceratometria média de 45,14 $\pm 1,95$ dioptrias, profundidade central média da câmara anterior de 2,49 $\pm 0,23 \mathrm{~mm}$; diâmetro axial médio de 21,82 $\pm 0,99$ milímetros, espessura média do cristalino de 4,82 \pm 0,30 milímetros, e a relação entre a espessura média do cristalino e diâmetro axial médio de 2,17 $\pm 0,19$.

Quando comparamos os dados ecobiométricos entre os olhos com GAP e os olhos contralaterais, observamos que não houve diferença estatisticamente significativa entre eles (Tabela 2).

\begin{tabular}{|lccc|}
\hline \multicolumn{4}{|l|}{ Tabela 1. Sexo, raça, olho com GAP, história familiar de glaucoma } \\
Sexo & $\mathbf{N}(\%)$ & $\mathbf{N}(\%)$ & Total $\mathbf{N}(\%)$ \\
& $14(70)$ & $6(30)$ & $20(100)$ \\
Raça & Feminino & Masculino & \\
& $12(60)$ & $8(40)$ & $20(100)$ \\
Olho com & Leucodérmicos & Feodérmicos \\
GAP & $11(55)$ & $9(45)$ \\
História familiar & Direito & Esquerdo \\
de glaucoma & $15(75)$ & $5(25)$ & $20(100)$ \\
GAP= glaucoma agudo primário & & \\
\hline
\end{tabular}

\section{DISCUSSÃO}

O GPAF apresenta uma morbidade elevada, principalmente em populações asiáticas, nas quais ocorre um elevado número de casos de cegueira em conseqüência dessa doença. Em estudo conduzido numa população urbana no sudeste da Índia, em 2000, verificou-se que $41,7 \%$ dos indivíduos com GPAF apresentaram cegueira uni ou bilateral ${ }^{(8)}$.

Acredita-se que a apresentação mais prevalente em caucasianos é a do GAP, ao contrário da forma crônica do GPAF que é mais freqüente em indivíduos de origem asiática e africana ${ }^{(4-5)}$. Essa última forma pode ser confundida com GPAA, devido a apresentação insidiosa, e sem crises agudas ${ }^{(4-5)}$. Como o GAP manifesta-se em ataques com fase sintomática aguda, a incidência mostra-se mais valiosa como um índice de morbidade ${ }^{(12)}$. Desse modo, analisamos a incidência do GAP no SGHSG, que é um serviço de referência no atendimento de pacientes com glaucoma no Estado de Minas Gerais, e identificamos 22,7 casos novos de GAP em 1000 atendimentos. Este resultado é um dado novo no Brasil. Quando comparamos as incidências obtidas nos estudos populacionais com a identificada no presente estudo, verificamos uma elevada incidência do GAP nesse trabalho ${ }^{(13-18)}$. Esse resultado justifica-se pelo fato de o SGHSG ser um serviço de referência no tratamento do glaucoma. Devemos salientar que o presente estudo avaliou a incidência de GAP em um serviço especializado em glaucoma, e desse modo, a base de análise epidemiológica não foi uma população de uma região, cidade, estado ou país, mas sim um grupo especifico de pacientes atendidos pelo SGHSG.

Estudos de incidência de GAP em serviços de referência são raros. Em estudo realizado no setor de glaucoma de um hospital terciário no norte da Índia, no período de janeiro/1995 a dezembro/1999, verificou que, dentre 2425 pacientes atendidos, 888 eram pacientes com GPAF $^{(22)}$. A forma crônica do GPAF era responsável por $576(64,86 \%)$ casos, $171(19,26 \%)$ eram da forma intermitente, e $141(15,88 \%)$ pacientes tiveram o diagnóstico de GAP $^{(22)}$. Desse modo, a incidência de GAP no serviço de glaucoma referido acima foi de 58,1 por 1000 atendimentos; valor superior ao encontrado no presente estudo, o que pode ser justificado pela maior frequiência de pacientes com ângulo fechado na Índia ${ }^{(8-9)}$.

A maior incidência e o maior risco relativo do GAP em mulheres no presente estudo estão de acordo com a literatu-

\begin{tabular}{|c|c|c|c|c|c|c|}
\hline & \multicolumn{2}{|c|}{ Olho afetado } & \multicolumn{2}{|c|}{ Olho contralateral } & \multirow[b]{2}{*}{$t$} & \multirow[b]{2}{*}{$p$} \\
\hline & Média & Desvio padrão & Média & Desvio padrão & & \\
\hline Ceratometria (dioptrias) & 45,14 & 1,95 & 44,97 & 2,03 & 1,829 & 0,083 \\
\hline PCCA (milímetros) & 2,49 & 0,23 & 2,53 & 0,23 & $-1,347$ & 0,194 \\
\hline AXL (milímetros) & 21,82 & 0,99 & 21,79 & 0,89 & 0,326 & 0,748 \\
\hline $\mathrm{CR}$ (milímetros) & 4,82 & 0,30 & 4,80 & 0,35 & 0,337 & 0,740 \\
\hline $\mathrm{CR} / \mathrm{AXL}$ & 2,17 & 0,19 & 2,20 & 0,14 & $-0,783$ & 0,443 \\
\hline
\end{tabular}


$\mathrm{ra}^{(13,16-18)}$. Devemos salientar a inexistência de casos de GAP em melanodérmicos no presente estudo, sendo que foi mais freqüente em leucodérmicos (60\%). De acordo com estudos realizados anteriormente, o GAP apresenta-se com elevada freqüência em caucasianos, enquanto que o GPAF crônico é mais prevalente em asiáticos ${ }^{(4-5)}$. Além disso, não verificamos casos de história familiar de GAP, apenas poucos casos $(25 \%)$ de GPAA na família, fato que não ocorre quando são analisados pacientes de GPAA, uma vez que a hereditariedade é um fator de risco importante nesses pacientes.

Quando analisamos comparativamente a ceratometria, a profundidade da câmara anterior, o diâmetro axial antero-posterior do olho, a espessura do cristalino e a relação entre a espessura do cristalino e o diâmetro axial entre os olhos com GAP e os olhos contralaterais, obtivemos resultados semelhantes em ambos os olhos, isto é, sem diferenças estatisticamente significativas, concordando com os de outros estu$\operatorname{dos}^{(19-21)}$. Isto reforça a possibilidade e, talvez, a probabilidade de ocorrer crise aguda no olho adelfo no decurso do tempo.

Alguns estudos documentaram a ocorrência de GAP no olho contralateral algum tempo após a eclosão da crise no olho inicialmente afetado ${ }^{(23-25)}$. Em trabalho realizado em 1962, constatou-se que, de 113 pacientes com GAP, 58 (51,3\%) apresentaram crise no olho contralateral, sendo que, em um terço desses pacientes, a nova crise ocorreu um ano após a primeira $^{(23)}$. Em 1977, relatou-se que $43(59,7 \%)$ de 72 pacientes não tratados com iridotomia a laser profilática desenvolveram $\mathrm{GAP}^{(24)}$. Em 1982 verificou-se que, de 245 pacientes com GAP, $25(10,2 \%)$ apresentaram crises bilaterais no momento do diagnóstico, e dos 220 restantes, 33 (15\%) pacientes desenvolveram crise no olho adelfo devido ao atraso de um mês para realização da profilaxia com laser ${ }^{(25)}$. Nenhum dos pacientes do presente estudo desenvolveu crise aguda de glaucoma no olho adelfo no período da pesquisa, uma vez que tratamento profilático era realizado o mais precocemente possível.

\section{CONCLUSÕES}

A incidência de GAP no SGHSG foi de 22,7 por 1000 atendimentos no período de um ano, sendo esta uma informação nova na literatura brasileira. Observamos que o GAP é mais freqüente em mulheres leucodérmicas, na faixa dos 60 anos e com história familiar negativa para glaucoma. Não tivemos GAP em pacientes melanodérmicos. A análise comparativa dos parâmetros ecobiométricos mostrou que eles são semelhantes no olho que sofreu a crise e no contralateral.

\section{ABSTRACT}

Purpose: To determine the incidence of the primary angleclosure glaucoma at the Glaucoma Service of São Geraldo Hospital, to establish the profile of these patients and to identify the possible risk factors. Methods: Transversal study.
Period of assessment: from September/2005 to August/2006. Inclusion criteria: diagnosis of primary angle-closure glaucoma. Exclusion criteria: presence of cataracts that cause low visual acuity or index-myopia, secondary glaucoma, plateau iris. Number of visits, incidence of primary angle-closure glaucoma, age, gender, race, family history of glaucoma, keratometric, and biometric data were assessed. Results: Of 879 attended patients, $20(2.3 \%)$ had the diagnosis of primary angle-closure glaucoma, therefore, the incidence of the primary angle-closure glaucoma was 22.7 cases per 1000 attended. Of those patients with primary angle-closure glaucoma: $6(30.0 \%)$ were men and $14(70.0 \%)$ women; the age varied from 40 to 73 years (average: $60.4 \pm 8.1$ years); $12(60.0 \%)$ were leukodermics and $8(40.0 \%)$ pheodermics; $5(25.0 \%)$ with positive familiar history of glaucoma. The relative risk for women was 1.44 (95\% IC). Eleven $(55.0 \%)$ patients had primary angle-closure glaucoma of the right eye and $9(45.0 \%)$ of the left. When biometric data and keratometry were compared between the affected and contralateral eye, no statistical significant difference was observed. Conclusions: The incidence of the primary angle-closure glaucoma at the Glaucoma Service of São Geraldo Hospital was 22.7 cases per 1000 attended. It was more frequent in leukodermic women, without family history of glaucoma and with an average age of 60.4 years. The affected and contralateral eyes were biometrically similar.

Keywords: Glaucoma, angle-closure/surgery; Glaucoma, angleclosure/epidemiology; Biometry; Risk factors; Comparative study

\section{REFERÊNCIAS}

1. Thylefors B, Negrel AD, Pararajasegaram R, Dadzie DY. Global data on blindness. Bull World Health Organ. 1995;73(1):115-21.

2. Thylefors B, Negrel AD. The global impact of glaucoma. Bull World Health Organ. 1994;72(3):323-6.

3. Yamamoto T, Iwase A, Araie M, Suzuki Y, Abe H, Shirato S, Kuwayana Y, Mishima HK, Shimizu H, Tomita G, Inoue Y, KitazawaY; Tajimi Study group, Japan Glaucoma Society. The Tajimi Study report 2: prevalence of primary angleclosure and secondary glaucoma in a Japanese population. Ophthalmology. 2005;112(10): 1661-9.

4. Bonomi L, Marchini G, Marraffa M, Bernardi P, De Franco I, Perfetti S, et al. Epidemiology of angle-closure glaucoma: prevalence, clinical types, and association with peripheral anterior chamber depth in the Egna-Neumarkt Glaucoma Study. Ophthalmology. 2000;107(5):998-1003. Comment in: Ophthalmology. 2001;108(9): 1514

5. Ramesh S, Maw C, Sutton CJ, Gandhewar JR, Kelly SP. Ethnic aspects of acute primary angle closure in a UK multicultural conurbation. Eye. 2005; 19(12):1271-5.

6. Foster PJ, Baasanhu J, Alsbirk PH, Munkhabayar D, Uranchimeg D, Johnson GJ. Glaucoma in Mongolia. A population-based survey in Hovsgol province, northern Mongolia. Arch Ophthalmol. 1996;114(10):1235-41. Comment in: Arch Ophthalmol. 1996;114(10):1251.

7. Foster PJ, Oen FT, Machin D, Ng TP, Devereux JG, Johnson GJ, et al. The prevalence of glaucoma in Chinese residents of Singapore: a cross-sectional population survey of the Tanjong Pagar district. Arch Ophthalmol. 2000; 118(8):1105-11.

8. Dandona L, Dandona R, Mandal P, Srinivas M, John RK, McCarty CA, et al. Angle-closure glaucoma in an urban population in southern India. The Andhra Pradesh eye disease study. Ophthalmology. 2000;107(9):1710-6.

9. Ramakrishnan R, Nirmalan PK, Krishnadas R, Thulasiraj RD, Tielsch JM, Katz J, et al. Glaucoma in a rural population of southern India: the Aravind comprehensive eye survey. Ophthalmology. 2003;110(8):1484-90. Erratum 
in: Ophthalmology. 2004;111(2):331. Comment in: Ophthalmology. 2004; 111(4):846; author reply 846-7.

10. Cedrone C, Culasso F, Cesareo M, Zapelloni A, Cedrone P, Cerulli L. Prevalence of glaucoma in Ponza, Italy: a comparison with other studies. Ophthalmic Epidemiol. 1997;4(2):59-72.

11. Wensor MD, McCarty CA, Stanislavsky YR, Livingston PM, Taylo HR. The prevalence of glaucoma in the Melbourne Visual Impairment Project. Ophthalmology. 1998;105(4):733-9.

12. Seah SK, Foster PJ, Chew PT, Jap A, Oen F, Fam HB, et al. Incidence of acute primary angle-closure glaucoma in Singapore. An island-wide survey. Arch Ophthalmol. 1997; 115(11):1436-40.

13. Teikari J, Raivio I, Nurminen M. Incidence of acute glaucoma in Finland from 1973 to 1982. Graefes Arch Clin Exp Ophthalmol. 1987;225(5):357-60.

14. Erie JC, Hodge DO, Gray DT. The incidence of primary angle-closure glaucoma in Olmsted County, Minnesota. Arch Ophthalmol. 1997;115(2):177-81.

15. Lai JS, Liu DT, Tham CC, Li RT, Lam DS. Epidemiology of acute primary angle-closure glaucoma in the Hong Kong Chinese population: prospective study. Hong Kong Med J. 2001;7(2):118-23.

16. Bojic L, Mandic Z, Ivanisevic M, Bucan K, Kovacevic S, Gverovic A, et al. Incidence of acute angle-closure glaucoma in Dalmatia, southern Croatia. Croat Med J. 2004;45(3):279-82. Comment in: Croat Med J. 2004;45(4):503-4; author reply $504-5$.
17. Ivanisevic M, Erceg M, Smoljanovic A, Trosic Z. The incidence and seasonal variations of acute primary angle-closure glaucoma. Coll Antropol. 2002; 26(1):41-5.

18. Vadot E, Grateau C. [The frequency of acute glaucoma crises. Implications for the detection of the risk of angle-closure]. Bull Soc Ophthalmol Fr. 1989; 89(5):675-7. French.

19. Calixto N, Cronemberger S. Glaucoma simples x glaucoma agudo. Estudo ecobiométrico. Arq Bras Oftalmol. 1986;49(1):1-8.

20. Tornquist R. Chamber depth in primary acute glaucoma. Br J Ophthalmol. 1956;40(7):421-9.

21. Yamane R. Relação entre a espessura do cristalino e o comprimento axial do olho no glaucoma agudo primário [tese]. Rio de Janeiro: Universidade Federal do Rio de Janeiro; 1994.

22. Das J, Bhomaj S, Chaudhuri Z, Sharma P, Negi A, Dasgupta A. Profile of glaucoma in a major eye hospital in north India. Indian J Ophthalmol. 2001; 49(1):25-30.

23. Lowe RF. Acute angle-closure glaucoma: the second eye: an analysis of 200 cases. Br J Ophthalmol. 1962;46(11):641-50.

24. Snow JT. Value of prophylactic peripheral iridectomy on the second eye in angle-closure glaucoma. Trans Ophthalmol Soc UK. 1977;97(1):189-91.

25. Edwards RS. Behaviour of the fellow eye in acute angle-closure glaucoma. $\mathrm{Br}$ J Ophthalmol. 1982;66(9):576-9. 